\title{
Downregulation of TrkA protein expression by miRNA-92a promotes the proliferation and migration of human neuroblastoma cells
}

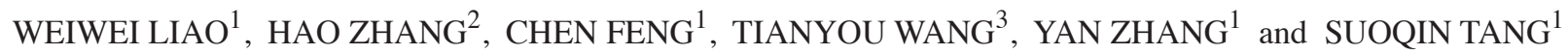 \\ ${ }^{1}$ Department of Pediatrics, Chinese PLA General Hospital, Beijing 100853; ${ }^{2}$ Department of Pediatrics, The Central Hospital \\ of China Aerospace Corporation, Beijing 100049; ${ }^{3}$ Department of Hematology-Oncology, Children's Hospital, \\ Capital Institute of Pediatrics, Beijing 100020, P.R. China
}

Received September 10, 2013; Accepted March 18, 2014

DOI: $10.3892 / \mathrm{mmr} .2014 .2235$

\begin{abstract}
The aims of this study were to investigate the regulation of TrkA protein by micro (mi)RNA-92a and its effect on the proliferation and migration of human neuroblastoma cells. The BE(2)-M17 human neuroblastoma cell line was cultured and transfected with either miRNA-92a mimics or miRNA-92a inhibitors. The expression levels of miRNA-92a and TrkA mRNA were detected by quantitative polymerase chain reaction prior and subsequent to transfection. TrkA protein was quantitatively detected by flow cytometry. The proliferation and migration of neuroblastoma cells were examined in vitro by Cell Counting Kit-8 and Transwell assays. Transfection of BE(2)-M17 cells with miRNA-92a mimics produced significantly higher expression levels of miRNA-92a compared with those in the same cells transfected with negative controls (NCs). Increased proliferation and migration of the cells was also observed. Transfection of BE(2)-M17 cells with miRNA-92a inhibitors resulted in significantly lower expression levels of miRNA-92a when compared with those of the same cells transfected with NCs $(\mathrm{P}<0.01)$. This reduction in the miRNA-92a expression levels was accompanied by reduced proliferation and migration of the cells. The expression levels of TrkA mRNA and protein after $24 \mathrm{~h}$ transfection with the miRNA-92a mimics were significantly reduced when compared with the control $(\mathrm{P}<0.01)$. However, the expression
\end{abstract}

Correspondence to: Dr Suoqin Tang, Department of Pediatrics, Chinese PLA General Hospital, 28 Fuxing Road, Beijing 100853, P.R. China

E-mail: suoqin@yahoo.com

Abbreviations: DMEM, Dulbecco's modified Eagle's medium; miRNA, microRNA; NGF, nerve growth factor; PBS, phosphate-buffered saline; qPCR, quantitative polymerase chain reaction; Trk, tyrosine kinase

Key words: neuroblastoma, miRNA-92a, TrkA protein, proliferation levels of TrkA were significantly higher $(\mathrm{P}<0.01)$ after $48 \mathrm{~h}$ transfection with miRNA-92a inhibitors when compared with the control. In conclusion, miRNA-92a promoted the proliferation and migration of human neuroblastoma cells through downregulation of TrkA, which suggested that miRNA-92a may be a potential target for human neuroblastoma treatment in the future.

\section{Introduction}

Neuroblastoma is the most common extracranial solid tumor of childhood and exhibits various clinical manifestations and diverse prognoses $(1,2)$. Certain tumor cells undergo spontaneous regression while others exhibit continuous progression. The prognosis of neuroblastoma is associated with the age of onset, the extent of disease and the biological characteristics of the tumor. MYCN gene amplification is an independent high risk factor for neuroblastoma. MYCN regulates various genes through multiple biochemical signaling pathways and thereby promotes the growth of malignant tumors (3). Previous studies have shown that the expression levels of the MYCN gene were associated with a variety of abnormal micro (mi)RNA regulation patterns (4-7). miRNAs are a class of endogenous non-coding RNAs 22 nt in length, which may inhibit the translation of specific target mRNAs and induce its degradation, thereby affecting cell proliferation, differentiation, apoptosis and other biological processes (8). Various miRNA molecules have been associated with neuroblastoma; one of the MYCN gene regulating factors in neuroblastoma is the miRNA17-92 cluster (9-11).

The tyrosine kinase (Trk) receptor family includes TrkA, TrkB and TrkC. Previous studies have shown that the Trk family is associated with the prognosis of neuroblastoma (12). Neuroblastoma with TrkA expression results in an improved prognosis, as TrkA combines with its ligand, nerve growth factor (NGF), to promote spontaneous regression or differentiation of the tumor. However, neuroblastoma with TrkB expression results in a poor prognosis due to amplification of the MYCN gene. TrkB ligands from neuroblastoma, via autocrine or paracrine survival pathways, may enhance the viability, drug resistance and angiogenesis of TrkB-expressing 
tumors (13). To the best of our knowledge, no studies have confirmed the association between the miRNA17-92 cluster and the TrK family. In the present study, the miRNA17-92 cluster regulated by MYCN gene was hypothesized to be associated with the TrK family and thus affect the prognosis of neuroblastoma. One of the miRNA members of miRNA17-92 cluster, miRNA-92a, was selected to investigate its effect on human neuroblastoma cells and the underlying mechanisms.

\section{Materials and methods}

Cell culture. Primary BE(2)-M17 human neuroblastoma cell lines with an amplified MYCN gene were purchased from the cell stores of the Chinese Academy of Medical Sciences (Beijing, China), and cultured in Dulbecco's modified Eagle's medium (DMEM; Hyclone Laboratories, Inc., Logan, UT, USA) supplemented with $10 \%$ fetal bovine serum (FBS; Gibco Life Technologies, Grand Island, NY, USA) and 1\% penicillin/streptomycin (Hyclone Laboratories, Inc.) at $37^{\circ} \mathrm{C}$ in a $5 \% \mathrm{CO}_{2}$ atmosphere.

Gene transfection. After 24 h of culture, the cells were starved in DMEM without 10\% FBS and 100 units penicillin/streptomycin, and divided into four groups. The appropriate quantity of the following reagents was added to each of the four groups respectively: miRNA-92a mimics negative control (NC) sense, 5'-UUCUCCGAACGUGUCACGUTT-3'; miRNA-92a mimicsNCantisense, 5'-ACGUGACACGUUCGGAGAATT-3'; miRNA-92a mimics sense, 5'-UAUUGCACUUGUCCCGGCC UGU-3'; miRNA-92amimicsantisense,5'-AGGCCGGGACAA GUGCAAUAUU-3'; miRNA-92a inhibitor NC sense, 5'-CAGUACUUUUGUGUA GUACAA-3'; and miRNA-92a inhibitors antisense, 5'-ACAGGCCGGGACAAGUGCA AUA-3' (all purchased from Gene Pharma, Shanghai, China). Lipofectamine 2000 (Invitrogen Life Technologies, Carlsbad, CA, USA) was added to the culture media of each of above groups and oligonucleotides were adjusted to a final concentration of $160 \mathrm{nM}$. All cells were incubated in DMEM containing $10 \% \mathrm{FBS}$ at $37^{\circ} \mathrm{C}$ in a humidified incubator with $5 \% \mathrm{CO}_{2}$. The miRNA-92a mimics and inhibitor were fluorescently labeled with fluorescein (Sigma-Aldrich, St. Louis, MO, USA) for verification of transfection.

Total RNA extraction. Total RNA from cultured cell lines was extracted at $0,24,48$ and $72 \mathrm{~h}$ post-transfection following the manufacturer's instructions of the RNAiso Plus reagent (Takara Bio Inc., Otsu, Japan). The concentration and quality of the RNA were determined with GeneQuan Pro (Biochrom Ltd., Cambridge, UK), with the OD260nm/OD280nm between 1.8 and 2.2 . The RNA was stored at $-80^{\circ} \mathrm{C}$.

Quantitative polymerase chain reaction ( $q P C R)$ for miRNA and TrkA mRNA expression. qPCR analysis for miRNA-92a was performed in triplicate with the One Step Primescript ${ }^{\circledR}$ miRNA cDNA Synthesis kit (Takara Bio Inc.) and SYBR ${ }^{\circledR}$ Premix Ex TaqTMII (Perfect Real Time; Takara Bio Inc.) according to the manufacturer's instructions. U6 small nuclear RNAs served as internal controls. The mixture was incubated for $30 \mathrm{sec}$ at $95^{\circ} \mathrm{C}$ for $1 \mathrm{cycle}$, followed by $5 \mathrm{sec}$ at $95^{\circ} \mathrm{C}$ and $30 \mathrm{sec}$ at $60^{\circ} \mathrm{C}$ for 40 cycles using the DNA Engine Opticon2
(MJ Research Inc. Waltham, MA). The fold-change in miRNA-92a expression levels was calculated using $\triangle \mathrm{CT}$ and $2^{-\triangle \Delta C T}$. The following primers were used respectively: miRNA-92a sense, 5'-TATTGCACTTGTCCCGGCCTG-3'; the miRNA-92a antisense strand was constructed using general primers; U6 sense, 5'-TCGCTTCGGCAGCACATA-3'; U6 antisense, 5'-TTGCGTGTCATCCTTGCG-3' (Sunbiotech Co. Ltd., Beijing, China).

TrkA mRNA expression levels were measured at 24 and $48 \mathrm{~h}$ post-transfection by qPCR, which was performed in triplicate using the same method as for miRNA-92a. $\beta$-actin served as the internal control. The following primers were used respectively: TrkA mRNA sense, 5'-TATTGCACTTGTCC CGGCCTG-3'; TrkA mRNA antisense, 5'-ACAAGGAGCAG CGTAGAAAGGA-3'; $\beta$-actin sense, 5'-TGACGTGGACATC CGCAAAG-3'; $\beta$-actin antisense, 5'-CTGGAAGGTGGA CAGCGAGG-3' (Sunbiotech Co. Ltd.).

Cell Counting Kit-8 (CCK-8) assay. The transfected neuroblastoma cells were seeded into 96 -well plates $\left(5.0 \times 10^{3}\right.$ cells/well) containing $100 \mu \mathrm{l}$ DMEM medium supplemented with $10 \%$ FBS. Cell viability was detected by CCK- 8 assay (Dojin Laboratories, Kumamoto, Japan) at 0, 24, 48 and $72 \mathrm{~h}$ post-transfection. The absorbance at $450 \mathrm{~nm}$ (A450) of each well was read on a Model 550 spectrophotometer (Bio-Rad, Hercules, CA, USA).

Transwell migration assay. Cellular migration was measured using a modification of the method as reported previously (14), using 24-well Transwell cell culture chambers filtered with multiporous polycarbonate membranes (Corning Inc., NY, USA). The BE(2)-M17 cells were cultured for $6 \mathrm{~h}$ in serum-free DMEM medium without antibiotics following transfection. Each group of cells was digested to form a cell suspension and adjusted to a density of either $2 \times 10^{5}$ cells per ml (miRNA-92a mimics and miRNA-92a mimics NC groups) or $3 \times 10^{5}$ cells per ml (miRNA-92a inhibitor and miRNA-92a inhibitor NC groups). A volume of $100 \mu \mathrm{l}$ cell suspension cultured in $500 \mu$ 1 DMEM medium with $10 \%$ FBS was extracted and plated onto a 24-well Transwell plate. The plates were placed in a humidified $5 \% \mathrm{CO}_{2}$ incubator for $36 \mathrm{~h}$ at $37^{\circ} \mathrm{C}$. The upper surface of the membrane was wiped with cotton swabs to remove non-migrated cells, and the remaining cells were fixed in $95 \%$ ethanol (Beijing Chemical Works, Beijing, China) for $10 \mathrm{~min}$ and then stained with $0.1 \%$ crystal violet (Sigma-Aldrich) for $15 \mathrm{~min}$. Digital images of cells were obtained using the DMI4000B DFC500 inverted microscope (Leica Microsystems AG, Wetzlar, Germany; magnification, $\mathrm{x} 200$ ). The number of cells in each image was counted by Scion Image software (Scion Corporation, Torrance, CA, USA). Each treatment in the migration assay was performed in triplicate.

Flow cytometry assay for TrkA protein expression. A total of $\sim 1.0 \times 10^{6}$ cells were fixed in phosphate-buffered saline (PBS) with $4 \%$ formaldehyde for $10 \mathrm{~min}$ at $37^{\circ} \mathrm{C}$ and subsequently incubated with the primary rabbit polyclonal anti-human TrkA antibody (Abcam Plc, Cambridge, UK) at $50 \mu \mathrm{g} / \mathrm{ml}$ for $1 \mathrm{~h}$. Following three washes with PBS, the cells were incubated with $2 \mu \mathrm{g} / \mathrm{ml}$ anti-rabbit IgG for $1 \mathrm{~h}$ (Cell Signaling Technology 


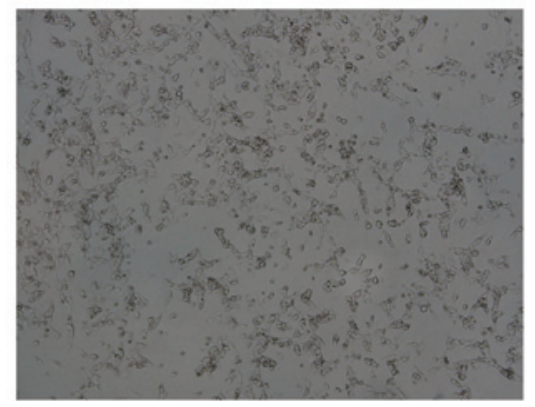

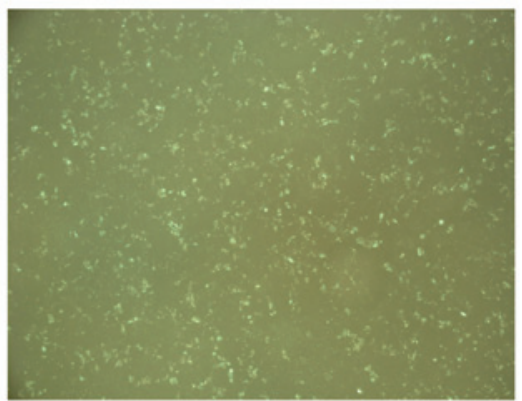

Figure 1. Cells transfected with the microRNA-92a inhibitor fluorescently labeled with fluorescein (A) under natural light and (B) under green fluorescence in the same field of vision. Magnification, x100.

Inc., Boston, MA, USA). The cells were resuspended in PBS and analyzed with the CytomicsTM FC500 flow cytometer (Beckman Coulter Inc. Fullerton, CA, USA).

Statistical analysis. All data were analyzed by SPSS software (version 13; SPSS, Inc., Chicago, IL, USA) and the significance of the difference between groups was determined by Student's t-test. $\mathrm{P}<0.05$ was considered to indicate a statistically significant difference.

\section{Results}

Effect of miRNA-92 a transfection and its changes in expression. In order to analyze the effect of miRNA-92a transfection and its expression, the solution of miRNA-92a mimics, miRNA-92a mimics NC, miRNA-92a inhibitors and miRNA-92a inhibitor $\mathrm{NC}$ was replaced $6 \mathrm{~h}$ later following the transfection. Tumor cells were focused under the fluorescence microscope at a magnification of $x 100$. The cells were observed under natural light and under fluorescence in the same field of vision (Fig. 1). Granular fluorescence was observed in a large number of the tumor cells which indicated miRNA transfection of the cells. To further verify transfection, total RNA at 24,48 and $72 \mathrm{~h}$ after transfection was isolated and qPCR was employed to detect the relative expression levels of miRNA-92a at three time points. The maximum miRNA-92a expression level following miRNA-92a mimics transfection was detected at $24 \mathrm{~h}$ after transfection, then gradually decreased from this level (Fig. 2A). The miRNA-92a expression levels were reduced in comparison with the NC group $24 \mathrm{~h}$ after miRNA-92a inhibitor transfection and the inhibition reached a peak at $48 \mathrm{~h}$, which lasted until $72 \mathrm{~h}$ post-transfection (Fig. 2B). These results demonstrate that the interference effect was significantly enhanced $24 \mathrm{~h}$ post-transfection with miRNA-92a mimics $(\mathrm{P}<0.01)$ and $48 \mathrm{~h}$ post-transfection with miRNA-92a inhibitors $(\mathrm{P}<0.01)$.

miRNA-92a promotes the proliferation of BE(2)-M17 human neuroblastoma cells. In order to investigate the functional role of miRNA-92a in neuroblastoma cells, the effect of miRNA-92a mimics and miRNA-92a inhibitors on the proliferation of BE(2)-M17 human neuroblastoma cell line was examined. The cells were transfected with either miRNA or NC for 24 , 48 and $72 \mathrm{~h}$. CCK-8 assay and direct cell count revealed that overexpression of miRNA-92a mimics significantly increased
A

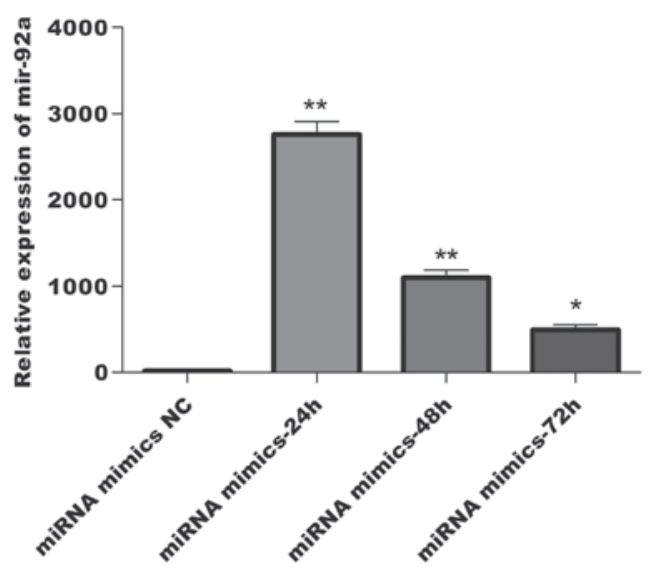

B

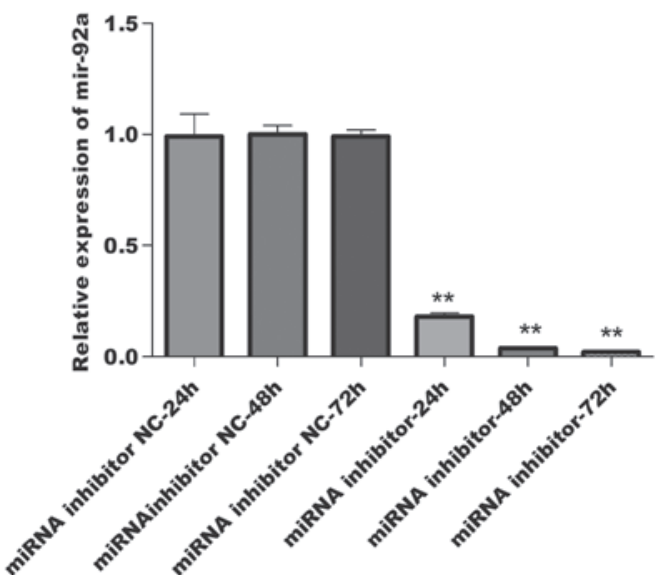

Figure 2. Quantitative polymerase chain reaction analysis of miRNA-92a at 24, 48 and $72 \mathrm{~h}$ after transfection. U6 served as an endogenous control. The expression levels of miRNA-92a are relative to the NC (set as 1.0). (A) The maximum expression level of miRNA-92a following transfection with the miRNA-92a mimics occurred at $24 \mathrm{~h}$ after transfection, but was then decreased from this level at 48 and $72 \mathrm{~h}$. (B) The miRNA-92a expression level was significantly reduced $24 \mathrm{~h}$ after transfection with the miRNA-92a inhibitor and was further decreased after $48 \mathrm{~h}$. After $72 \mathrm{~h}$ the expression levels remained in decline but this decline had markedly slowed down. Data are presented as the mean \pm standard deviation $(\mathrm{n}=3)$. ${ }^{*} \mathrm{P}<0.05$ and ${ }^{* * *} \mathrm{P}<0.01$ vs. the respective NC group. miRNA, microRNA; NC, negative control.

the proliferation of $\mathrm{BE}(2)-\mathrm{M} 17$ human neuroblastoma cells and overexpression of miRNA-92a inhibitors significantly inhibited the proliferation of neuroblastoma cells (Fig. 3). 
Table I. Average fluorescence intensity of Trk protein by fluorescence-activated cell sorting.

\begin{tabular}{lcccc}
\hline Group & $\begin{array}{c}\text { miRNA-92a } \\
\text { mimics NC }\end{array}$ & $\begin{array}{c}\text { miRNA-92a } \\
\text { mimics }\end{array}$ & $\begin{array}{c}\text { miRNA-92a } \\
\text { inhibitors NC }\end{array}$ & $\begin{array}{c}\text { miRNA-92a } \\
\text { inhibitors }\end{array}$ \\
\hline Average fluorescence intensity at $24 \mathrm{~h}$ & $31.26 \pm 0.26$ & $29.45 \pm 0.36^{\mathrm{a}}$ & $28.05 \pm 0.43$ & $26.72 \pm 0.99$ \\
Average fluorescence intensity at $48 \mathrm{~h}$ & $25.12 \pm 0.76$ & $24.25 \pm 0.28$ & $22.42 \pm 0.86$ & $26.51 \pm 0.36^{\mathrm{a}}$ \\
\hline
\end{tabular}

Flow cytometry assay of TrkA protein expression levels. The average fluorescence value of the miRNA-92a mimics transfection group was significantly reduced at $24 \mathrm{~h}$ compared with the miRNA-92a mimics NC, while the average fluorescence value of the miRNA-92a inhibitors transfection group was significantly increased at $48 \mathrm{~h}$ compared with the miRNA-92a inhibitors NC. Results are presented as the mean \pm standard deviation. $(\mathrm{n}=3)$. ${ }^{\mathrm{P}}<0.01$. Trk, tyrosine kinase; miRNA, microRNA; NC, negative control.

Effect of miRNA-92a on BE(2)-M17 human neuroblastoma cell migration. To reveal whether miRNA-92a was involved in the regulation of migration of neuroblastoma cells, a Transwell migration assay was conducted. The number of cells across the membrane in the miRNA mimics transfected group (32.4 \pm 3.7$)$ was increased by $37.3 \%$ compared with that in the control group $(23.6 \pm 2.1)$, which was significantly different $(\mathrm{P}<0.05)$ (Fig. 4A and 4B). The number of cells across the membrane in the group of cells transfected with the miRNA-92a inhibitors $(21.3 \pm 2.3)$ was decreased by $40.8 \%$ compared with that in the control group (36.0 \pm 7.5$)$ which was also significantly different $(\mathrm{P}<0.05$, Fig. $4 \mathrm{C}$ and D). These data indicate that transfection with miRNA mimics was capable of enhancing the migration of cells, while transfection with miRNA inhibitors reduced the migration of cells.

TrkA expression levels are inversely correlated with the miRNA-92 a expression levels. In view of the established role of miRNA-92a as an effector for indirect MYCN-induced downregulation of protein-coding genes, it was hypothesized that this miRNA may regulate TrkA. To investigate this possibility, TrkA mRNA expression following conditional up- or downregulation of miRNA-92a was verified in BE(2)-M17 human neuroblastoma cell lines. Total RNA was extracted at 24 and 48 h post-transfection and qPCR was used to detect TrkA mRNA expression at these two time points; the TrkA protein expression levels were detected by flow cytometry. The data from the qPCR and flow cytometry measurements revealed that the TrkA mRNA expression levels (Fig. 5) and the average fluorescence value (Table I) at $24 \mathrm{~h}$ post-transfection were significantly reduced in the miRNA-92a mimics transfection group compared with the control group, although no statistical difference was detected at $48 \mathrm{~h}$. However, when the miRNA-92a inhibitor transfection group was compared with its control, the TrkA mRNA expression levels and the average fluorescence value at $48 \mathrm{~h}$ post-transfection were significantly reduced, although no statistical difference was identified at $24 \mathrm{~h}$. These results demonstrated that the expression levels of TrkA protein in the cells transfected with miRNA-92a mimics were significantly reduced $(5.8 \%$ decrease; $\mathrm{P}<0.01)$ at $24 \mathrm{~h}$ post-transfection compared with the cells in the control group, while the expression levels of TrkA protein in the cells transfected with miRNA-92a inhibitor were significantly elevated $(18.2 \%$ increase; $\mathrm{P}<0.01)$ at $48 \mathrm{~h}$ post-transfection compared with the cells in the control
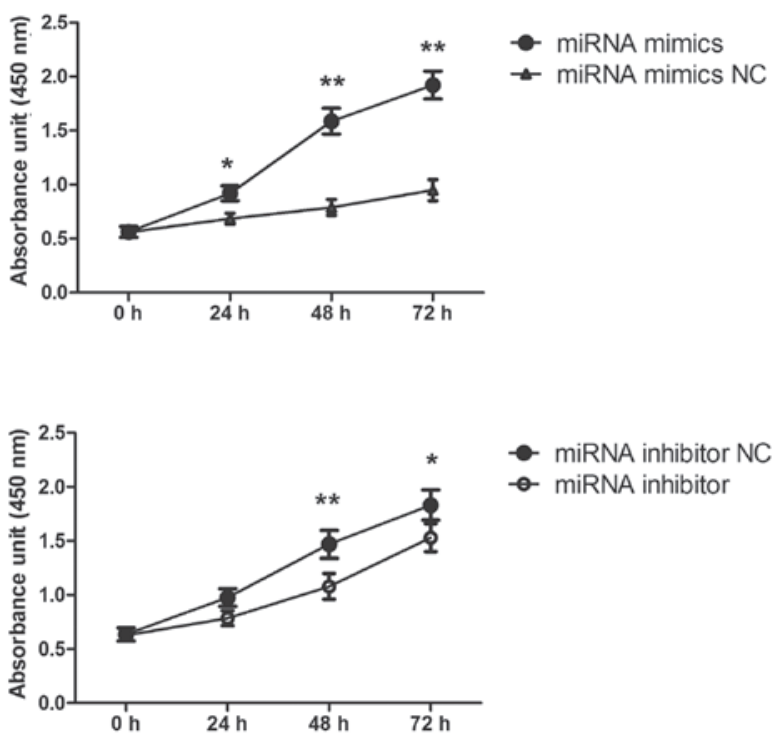

Figure 3. Cell Counting Kit-8 assay and direct cell count revealed that overexpression of miRNA-92a mimics significantly increased the proliferation of BE(2)-M17 human neuroblastoma cells, and overexpression of miRNA-92a inhibitors significantly inhibited the proliferation of the neuroblastoma cells. Data are presented as the mean \pm standard deviation $(n=6) .{ }^{*} \mathrm{P}<0.05$ and ${ }^{* *} \mathrm{P}<0.01$, vs. the respective NC. miRNA, microRNA; NC, negative control.

group. Overall, the miRNA-92a inhibitor exhibited a greater effect on TrkA protein than the miRNA-92a mimics.

\section{Discussion}

miRNAs are large groups of gene regulatory molecules which influence a number of gene encoding proteins. There is abundant evidence revealing $>1,400$ types of miRNA important in the pathogenesis of human diseases. One study has shown that miRNA inhibits the dedifferentiation and plasticity of cells in the process of tumor formation through the regulation of protein expression and the cell differentiation process. Furthermore, miRNA affects the cyclical adjustment of tumor cells, the integrity of the genomes, the stress response, apoptosis and metastasis (15).

The miRNA genes are located in introns or non-coding regions of the chromosome and are first transcribed as primary transcripts of 500-3,000 nt termed pri-miRNA. These molecules are then cut to 70 -bp miRNA precursors and then 

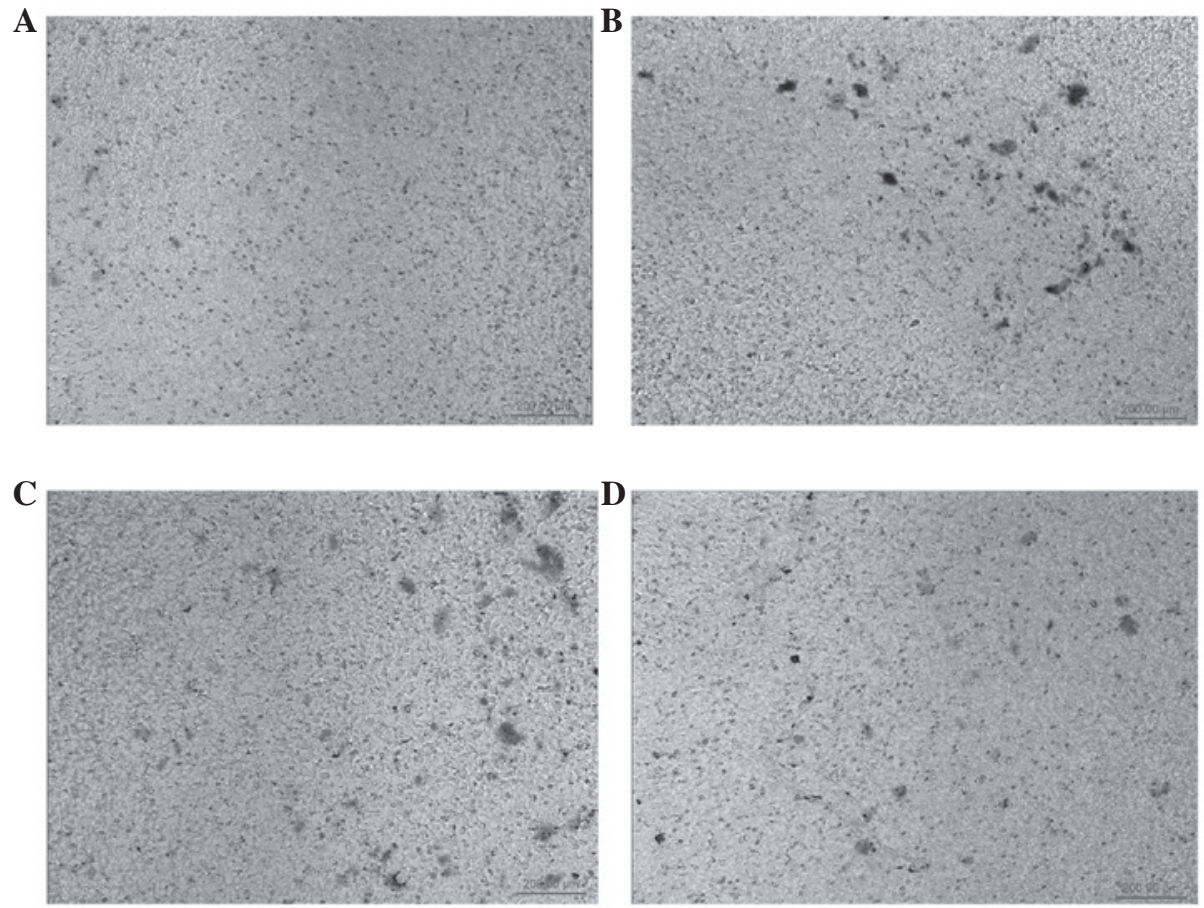

Figure 4. Transwell migration assay. Compared with the miRNA-92a mimics negative control group (A), the miRNA-92a mimics-transfected group (B) exhibited a significantly increased number of cells located across the membrane $(\mathrm{P}<0.05)$. Compared with the miRNA-92a inhibitors control group $(\mathrm{C})$ the miRNA-92a inhibitors transfected group (D) exhibited a significantly reduced number of cells across the membrane $(\mathrm{P}<0.05)$. Magnification, x200. miRNA, microRNA.

to 22 -bp mature miRNA assembled into RNA-induced silencing complexes to perform RNA interference gene silencing. When the miRNA molecule and the 3'UTR nucleotide of the target mRNA are complementary, the complex may inhibit the translation of the mRNA to protein and may induce the degradation of target mRNA $(8,16)$.

In the present study, double-stranded miRNA mimics, which have greater stability than single stranded ones, were selected to increase miRNA-92a expression levels. The miRNA-92a expression levels significantly increased $(\mathrm{P}<0.01)$ with miRNA mimics compared to an NC, achieving a peak $24 \mathrm{~h}$ after transfection and then gradually decreasing at 48 and $72 \mathrm{~h}$ after transfection. The miRNA-92a expression levels were found to progressively decrease compared with the NC following transfection with miRNA inhibitors. The effective regulation of miRNA-92a expression by transfection indicated that the research was reliable.

Studies have revealed that miRNA may affect a multitude of target genes and that one target gene may be regulated by multiple miRNA molecules (17-19). The miR-17-92 cluster has been considered to be the most effective carcinogenic miRNA, and hundreds of target genes of this cluster have been reported (20-23). One study observed a clear reduction in the expression levels of endogenous p $21 \mathrm{mRNA}$ and protein in SK-N-AS 17-5p cluster cells, as well as in SK-N-AS cells transiently transfected with miR-17-5p, although not in those transfected with miRNA-92 (21). Another study demonstrated that miRNA-17-92 is a potent inhibitor of transforming growth factor (TGF)- $\beta$ signaling. By functioning upstream and downstream of pSMAD2, miRNA-17-92 activation triggers downregulation of multiple key effectors along the TGF- $\beta$ signaling cascade as well as direct inhibition of the

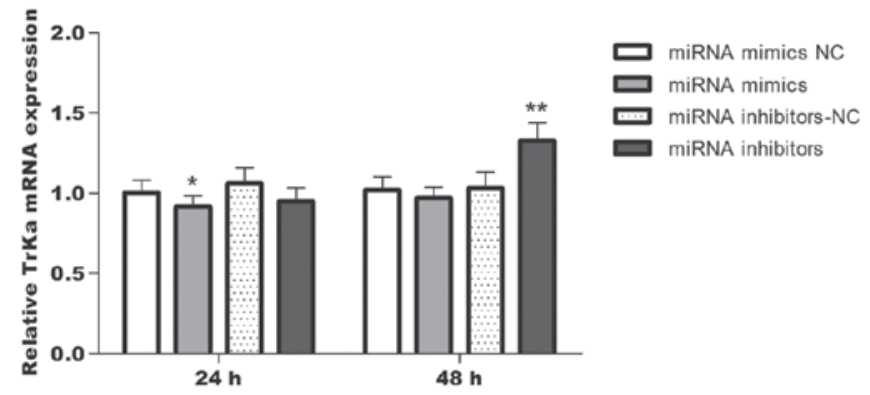

Figure 5. Quantitative polymerase chain reaction analysis of TrkA mRNA at 24 and $48 \mathrm{~h}$ after transfection. $\beta$-actin served as an endogenous control. Expression levels of TrkA mRNA are relative to the NC (set as 1.0). The TrkA mRNA of the miRNA-92a mimic transfection group was significantly reduced at $24 \mathrm{~h}$, while the TrkA mRNA of the miRNA-92a inhibitor transfection group was significantly increased at $48 \mathrm{~h}$. Results are expressed as the mean \pm standard deviation $(\mathrm{n}=3) .{ }^{*} \mathrm{P}<0.05$ and ${ }^{* *} \mathrm{P}<0.01$, vs. the respective $\mathrm{NC}$. miRNA, microRNA; NC, negative control.

TGF- $\beta$-responsive gene, but miRNA-92a did not affect the luciferase signals of SMAD2 (9). This suggests that every member of the miRNA-17-92 cluster exhibits its own characteristics, and the signaling pathway and mechanism of miRNA-92a may be different from the miRNA-17-92 cluster, although this requires further investigation.

Neuroblastoma cells have been observed to undergo differentiation and dissipation when TrkA is highly expressed, while cells with low TrkA expression levels exhibit the opposite behavior and become more invasive (3). In the present study, the proliferation and migration of neuroblastoma cells were found to be enhanced when TrkA expression was reduced, while the proliferation and migration 
capacity decreased when the TrkA expression was increased in the neuroblastoma cells.

Numerous studies have shown that high expression of TrkA in neuroblastoma tissue is associated with improved clinical characteristics in patients and lower MYCN gene amplification, while patients in the advanced stages of neuroblastoma usually exhibit lower expression levels of TrkA and the tumors cannot be treated with NGF to inhibit differentiation (24-26). The NGF/TrkA signaling pathway is important in promoting neuroblastoma differentiation and natural regression $(13,27,28)$. High expression of TrkA protein in neuroblastoma cells is associated with differentiation and regression (28-30), while lower TrkA expression is associated with increased invasiveness. One study suggested that activated Ras in the NGF/TrkA signaling pathway stimulates nuclear translocation of p53 and induces growth arrest by induction of p21WAF1 in PC12 cells (31). Activation of TrkA induces the phosphorylation and activation of SHC, phoshoinositide 3-kinase and phospholipase $C \gamma 1$, which are the primary effectors of Trk activity in NGF-treated PC12 cells (32). Ras/MAPK and AKT are activated downstream of these signaling pathways. Ras sequentially activates a series of kinases, including RAF1, mitogen-activated protein kinase kinase, mitogen-activated protein kinase (MAPK) and ribosomal S6 kinase (RSK) (13). MAPK and RSK translocate to the nucleus to initiate the activation of transcription factors that regulate NGF-inducible genes, leading to survival and neuronal differentiation. Other signaling proteins important for normal biological responses to ligand binding include SH2B/APS, fibroblast growth factor receptor substrate 2 and AKT (32). Further studies may be conducted to investigate whether miRNA-92a regulates the Ras/MAPK signaling pathway.

In the present study, transfection with miRNA-92a inhibitors was found to exert a more marked impact on TrkA protein expression levels than transfection with miRNA-92a mimics. Since the BE(2)-M17 neuroblastoma cell line exhibits MYCN gene amplification, the cell line was hypothesized to have high expression levels of miRNA-92a. Therefore, reducing the expression of miRNA-92a is more efficient than increasing the expression. Furthermore, the expression levels of the target protein were found to be reduced at $24 \mathrm{~h}$ after transfection with the miRNA-92a mimics, although these were restored to the levels of the NC after $48 \mathrm{~h}$. This may be considered to be an intracellular adjustment mechanism to correct target protein expression back to normal levels.

Although an association between the expression levels of miRNA-92a and TrkA has been observed, the mechanism and signaling pathway of TrkA expression regulated by miRNA-92a remains unclear. The present study has limitations in that there is not a great quantity of data and the findings have not been replicated in other neuroblastoma cell lines. If a particular solution were found to inhibit or restore the TrkA signaling pathway when the study is repeated, the results may be more convincing.

In conclusion, the present study demonstrated that the biological behavior of neuroblastoma cells was markedly altered when the expression levels of miRNA-92a were elevated or reduced. The proliferation and migration capacity of neuroblastoma cells exhibited a positive correlation with the expression levels of miRNA-92a in tumor cells, and a negative correlation with TrkA protein expression levels. miRNA-92a may affect neuroblastoma cell proliferation and migration capacity by regulating TrkA protein expression levels. This may provide an experimental basis for the treatment of neuroblastoma with miRNA-92a.

\section{Acknowledgements}

This study was supported by the China National 863 Program (grant no. 2012AA020804).

\section{References}

1. Maris JM, Mosse YP, Bradfield JP, et al: Chromosome 6p22 locus associated with clinically aggressive neuroblastoma. N Engl J Med 358: 2585-2593, 2008.

2. Schwab M, Westermann F, Hero B and Berthold F Neuroblastoma: biology and molecular and chromosomal pathology. Lancet Oncol 4: 472-480, 2003

3. Alaminos M, Mora J, Cheung NK, et al: Genome-wide analysis of gene expression associated with MYCN in human neuroblastoma. Cancer Res 63: 4538-4546, 2003.

4. Mestdagh P, Fredlund E, Pattyn F, et al: MYCN/c-MYC-induced microRNAs repress coding gene networks associated with poor outcome in MYCN/c-MYC-activated tumors. Oncogene 29: 1394-1404, 2010.

5. O'Donnell KA, Wentzel EA, Zeller KI, Dang CV and Mendell JT: c-Myc-regulated microRNAs modulate E2F1 expression. Nature 435: 839-843, 2005

6. MaL, Young J,PrabhalaH, et al: miR-9, a MYC/MYCN-activated microRNA, regulates E-cadherin and cancer metastasis. Nat Cell Biol 12: 247-256, 2010.

7. Welch C, Chen Y and Stallings RL: MicroRNA-34a functions as a potential tumor suppressor by inducing apoptosis in neuroblastoma cells. Oncogene 26: 5017-5022, 2007.

8. Bartel DP: MicroRNAs: genomics, biogenesis, mechanism, and function. Cell 116: 281-297, 2004.

9. Mestdagh P, Boström AK, Impens F, et al: The miR-17-92 microRNA cluster regulates multiple components of the TGF-beta pathway in neuroblastoma. Mol Cell 40: 762-773, 2010.

10. Lovén J, Zinin N, Wahlström T, et al: MYCN-regulated microRNAs repress estrogen receptor-alpha (ESR1) expression and neuronal differentiation in human neuroblastoma. Proc Natl Acad Sci USA 107: 1553-1558, 2010.

11. Nara K, Kusafuka T, Yoneda A, Oue T, Sangkhathat S and Fukuzawa M: Silencing of MYCN by RNA interference induces growth inhibition, apoptotic activity and cell differentiation in a neuroblastoma cell line with MYCN amplification. Int J Oncol 30: 1189-1196, 2007.

12. Kogner P, Barbany G, Dominici C, Castello MA, Raschellá G and Persson $\mathrm{H}$ : Coexpression of messenger RNA for TRK protooncogene and low affinity nerve growth factor receptor in neuroblastoma with favorable prognosis. Cancer Res 53: 2044-2050, 1993.

13. Brodeur GM, Minturn JE, Ho R, et al: Trk receptor expression and inhibition in neuroblastomas. Clin Cancer Res 15: 3244-3250, 2009.

14. Valster A, Tran NL, Nakada M, Berens ME, Chan AY and Symons M: Cell migration and invasion assays. Methods 37: 208-215, 2005.

15. Negrini M, Nicoloso MS and Calin GA: MicroRNAs and cancer - new paradigms in molecular oncology. Curr Opin Cell Biol 21: 470-479, 2009.

16. Woo CW, Tan F, Cassano H, Lee J, Lee KC and Thiele CJ: Use of RNA interference to elucidate the effect of MYCN on cell cycle in neuroblastoma. Pediatr Blood Cancer 50: 208-212, 2008.

17. Krek A, Grün D, Poy MN, et al: Combinatorial microRNA target predictions. Nat Genet 37: 495-500, 2005

18. Bang-Berthelsen CH, Pedersen L, Fløyel T, Hagedorn PH, Gylvin $\mathrm{T}$ and Pociot F: Independent component and pathway-based analysis of miRNA-regulated gene expression in a model of type 1 diabetes. BMC Genomics 12: 97, 2011. 
19. Xie SY, Li YJ, Wang PY, Jiao F, Zhang S and Zhang WJ: miRNA-regulated expression of oncogenes and tumor suppressor genes in the cisplatin-inhibited growth of K562 cells. Oncol Rep 23: 1693-1700, 2010.

20. Olive V, Jiang I and He L: mir-17-92, a cluster of miRNAs in the midst of the cancer network. Int J Biochem Cell Biol 42: 1348-1354, 2010.

21. Fontana L, Fiori ME, Albini S, et al: Antagomir-17-5p abolishes the growth of therapy-resistant neuroblastoma through p21 and BIM. PLoS One 3: e2236, 2008.

22. Fontana L, Pelosi E, Greco P, et al: MicroRNAs 17-5p-20a-106a control monocytopoiesis through AML1 targeting and M-CSF receptor upregulation. Nat Cell Biol 9: 775-787, 2007.

23. Haug BH, Henriksen JR, Buechner J, et al: MYCN-regulated miRNA-92 inhibits secretion of the tumor suppressor DICKKOPF-3 (DKK3) in neuroblastoma. Carcinogenesis 32: 1005-1012, 2011.

24. Westermark UK, Wilhelm M, Frenzel A and Henriksson MA: The MYCN oncogene and differentiation in neuroblastoma. Semin Cancer Biol 21: 256-266, 2011.

25. Peterson $S$ and Bogenmann E: The RET and TRKA pathways collaborate to regulate neuroblastoma differentiation. Oncogene 23: 213-225, 2004.
26. Nakagawara A: Molecular basis of spontaneous regression of neuroblastoma: role of neurotrophic signals and genetic abnormalities. Hum Cell 11: 115-124, 1998.

27. Nakagawara A, Arima M, Azar CG, Scavarda NJ and Brodeur GM: Inverse relationship between trk expression and $\mathrm{N}$-myc amplification in human neuroblastomas. Cancer Res 52: 1364-1368, 1992.

28. Kogner P, Barbany G, Dominici C, Castello MA, Raschellá G and Persson $\mathrm{H}$ : Coexpression of messenger RNA for TRK protooncogene and low affinity nerve growth factor receptor in neuroblastoma with favorable prognosis. Cancer Res 53: 2044-2050, 1993.

29. Nakagawara A: Trk receptor tyrosine kinases: a bridge between cancer and neural development. Cancer Lett 169: 107-114, 2001.

30. Nakagawara A: The NGF story and neuroblastoma. Med Pediatr Oncol 31: 113-115, 1998

31. Hughes AL, Gollapudi L, Sladek TL and Neet KE: Mediation of nerve growth factor-driven cell cycle arrest in PC12 cells by p53. Simultaneous differentiation and proliferation subsequent to p53 functional inactivation. J Biol Chem 275: 37829-37837, 2000.

32. Kaplan DR and Miller FD: Signal transduction by the neurotrophin receptors. Curr Opin Cell Biol 9: 213-221, 1997. 